\title{
PANBESY-INDUCED CORONARY VASOSPASM
}

Singapore Med J 2017; 58(9): 569-570 doi: 10.11622/smedj.2017014

Dear Sir,

Acute coronary syndrome (ACS) in young patients with no prior cardiovascular risk factors is rare and often found to be related to coronary anomalies or drug-induced coronary vasospasm. Among Asians, the Japanese have been reported to have a higher incidence of coronary vasospasm (40\%). ${ }^{(1)}$ Although there is currently no published global database to define the magnitude of drug-induced coronary vasospasm in young people, it is still an important cause of ACS and arrhythmia. ${ }^{(1)}$ Herein, we report a case of drug-induced coronary vasospasm in a young patient.

A 29-year-old woman presented with retrosternal chest tightness radiating to the back, which lasted for two hours and was associated with breathlessness, diaphoresis and palpitations. She was a non-smoker and had no family history of premature coronary artery disease. She reported taking a 15-mg dose of Panbesy (phentermine hydrochloride) daily for the last five months for weight loss, with no intentional overdose. She was non-obese (weight $60 \mathrm{~kg}$ ) and nondiabetic (haemoglobin A1c 5.1\%; fasting glucose $5.8 \mathrm{mmol} / \mathrm{L}$ ), and her lipid panel was as follows: total cholesterol $4.56 \mathrm{mmol} / \mathrm{L}$; low-density lipoprotein cholesterol $3.14 \mathrm{mmol} / \mathrm{L}$; triglycerides $0.51 \mathrm{mmol} / \mathrm{L}$; and high-density lipoprotein cholesterol $1.19 \mathrm{mmol} / \mathrm{L}$.

On examination, the patient showed signs of distress due to her ongoing angina. Vital signs showed regular heart rate, blood pressure and respiratory rate (98 beats/minute, $112 / 79 \mathrm{mmHg}$ and 20 breaths/minute, respectively). Systemic examination was unremarkable. Electrocardiography (ECG) showed sinus tachycardia with diffuse ST-segment depressions in the limb and chest leads, and ST-segment elevation in aVR (Fig. 1). Chest radiography was normal, computed tomography of the aorta showed no evidence of aortic dissection or intramural haematoma, and her D-dimer test result was also normal. However, her cardiac troponin levels were elevated (479 ng/L). Two-dimensional transthoracic echocardiography showed a left ventricular ejection fraction of $45 \%$, normal valves, and regional wall motion abnormalities in the basal, mid-inferior, mid-inferolateral, mid-anterolateral and basal-inferoseptal territories, which suggested ischaemia in the territories supplied by the right coronary artery and left circumflex artery. Subsequent coronary angiography, however, showed normal coronaries. No provocative test was performed.

The clinical impression was non-ST-segment elevation ACS resulting from coronary vasospasm, which was likely precipitated by Panbesy. The patient made an uneventful recovery and was advised to stop taking Panbesy. Her ECG was normal prior to discharge (Fig. 2).

Coronary vasospasm is a transient, intense vasoconstriction of the epicardial coronary arteries. It can either be unifocal (i.e. involving a focal segment of a single vessel) or multifocal (i.e. involving multiple segments of the same vessel or many vessels). Coronary vasospasm is commonly encountered in normal coronary arteries. ${ }^{(2)}$ Prinzmetal angina, also known as variant angina, is an entity characterised by rest angina and transient ST-T abnormalities due to coronary vasospasm. The mechanism of this condition is believed to involve endothelial dysfunction, although the coronaries are normal. Prinzmetal angina is confirmed by demonstrating coronary

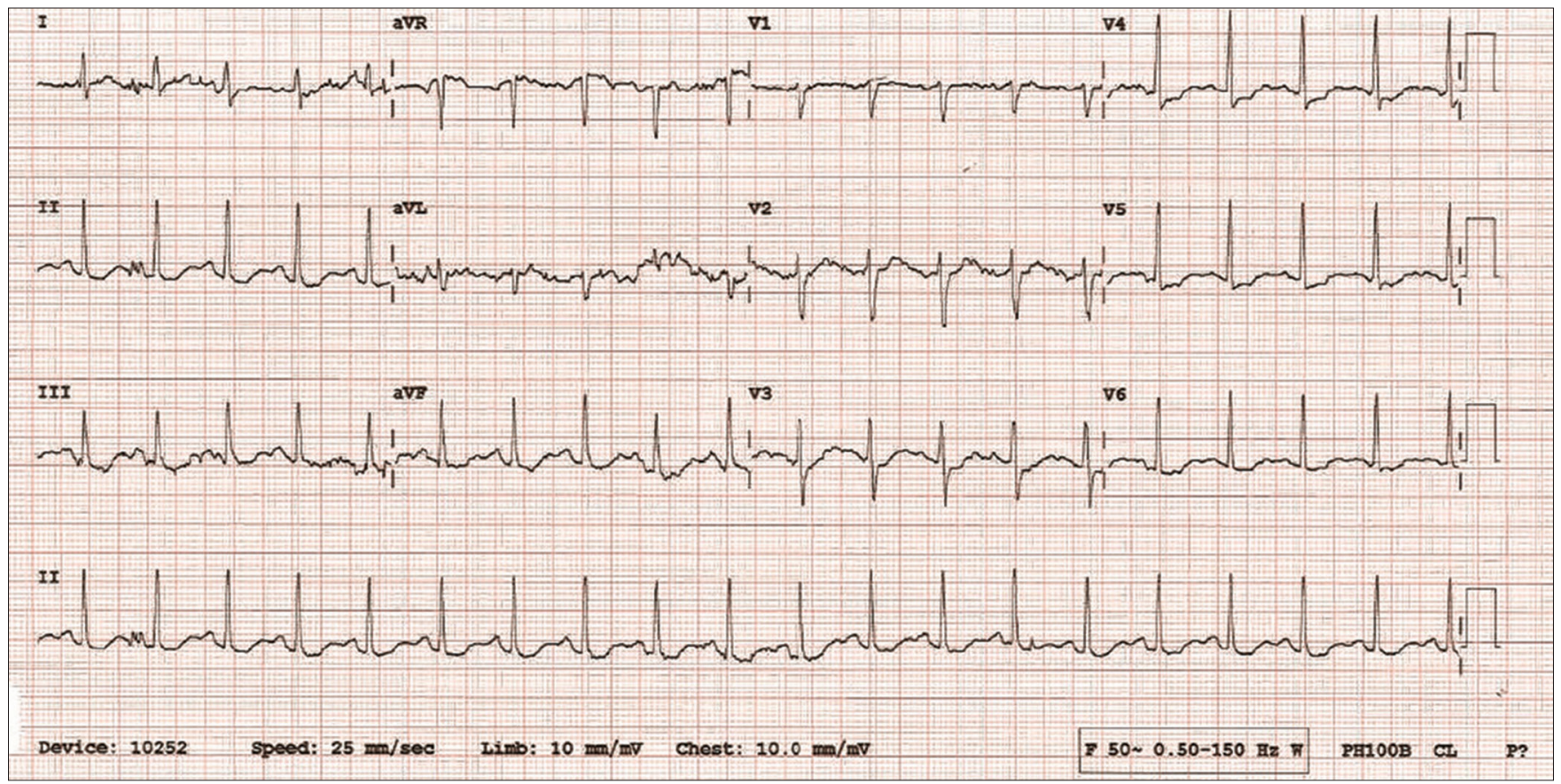

Fig. 1 12-lead ECG on presentation shows sinus tachycardia with diffuse ST-segment depressions in the limb and chest leads, and ST-segment elevation in aVR. 


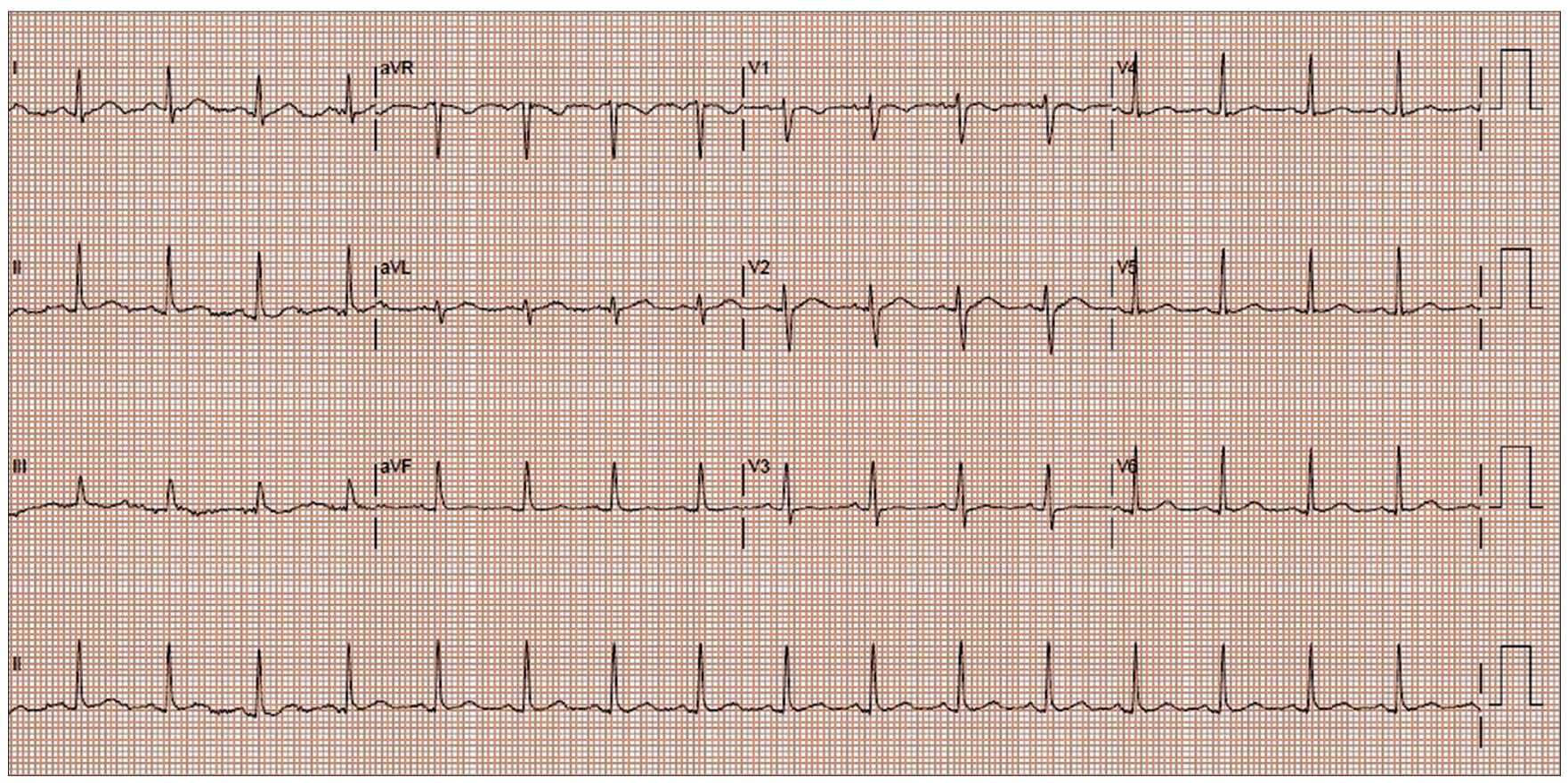

Fig. 2 12-lead ECG prior to discharge shows normal sinus rhythm.

artery spasm using provocative testing with either acetylcholine or ergonovine during coronary angiography. It can be diagnosed only in the absence of coronary artery disease and following a thorough history to exclude any drug-induced coronary vasospasm.

Panbesy, an anorectic agent, is a centrally acting sympathomimetic amine used in the treatment of obesity. Peripherally, it stimulates the release of neurotransmitters such as adrenaline. ${ }^{(3)}$ Cardiac complications associated with Panbesy include hypertension, coronary vasospasm leading to myocardial ischaemia/infarction and fatal arrhythmias such as ventricular fibrillation. ${ }^{(4)}$ However, very few randomised controlled trials have studied the safety of Panbesy. In Singapore, phentermine is marketed as Panbesy and Duromine, and is approved as a short-term adjunct for obesity treatment. The Ministry of Health, Singapore, has advised doctors to use the drug judiciously and monitor for side effects. ${ }^{(5)}$ To our knowledge at the time of writing, no cases of phentermine-induced ACS have been reported in Singapore. Thus, it is important to take a thorough drug history for young adults who present with ACS.

Yours sincerely,

Mohammed Rizwan Amanullah'1 ${ }^{1}$ Khim Leng Tong $^{2}$

${ }^{1}$ Department of Cardiology, National Heart Centre Singapore, ${ }^{2}$ Department of Cardiology, Changi General Hospital, Singapore. mohammed.rizwan@singhealth.com.sg

\section{RefERENCES}

1. El Menyar AA. Drug-induced myocardial infarction secondary to coronary artery spasm in teenagers and young adults. J Postgrad Med 2006; 52:51-6.

2. Lanza GA, Careri G, Crea F. Mechanisms of coronary artery spasm. Circulation 2011; 124:1774-82.

3. National Center for Biotechnology Information. Phentermine. In: PubChem Open Chemistry Database [online]. Available at: https://pubchem.ncbi.nlm.nih.gov/ compound/4771. Accessed March 6, 2016.

4. Tobbia P, Norris LA, Klima LD. Ventricular fibrillation coinciding with phentermine initiation. BMJ Case Rep 2012; 2012.

5. Ministry of Health, Singapore. Advisory on the use of phentermine for the purpose of weight management. 2009 Feb 23. Notification no. MH 71:25/3. Available at: https://www.moh.gov.sg/content/dam/moh_web/Publications/Guidelines/Other\%20Guidelines/2009/phentermine\%20advisory\%20final.pdf. Accessed March 6, 2016. 